\title{
An Efficient Vertex Addition Method for Broadcast Networks
}

\author{
Hovhannes A. Harutyunyan
}

Abstract. Broadcasting is a basic problem of communication in usual networks. Many papers have investigated the construction of minimum broadcast networks, the cheapest possible broadcast network architecture (having the fewest communication lines), in which broadcasting can be accomplished as fast as theoretically possible from any vertex. Since this problem is very difficult, numerous papers have investigated ways to construct sparse networks in which broadcasting can be completed in minimum time from any originator. In this paper we introduce an efficient vertex addition method that allows us to construct sparse networks and improve the best known upper bounds on the broadcast function $B(n)$ for many odd values of $n$. We also give the exact value of $B(127)$.

\section{Introduction}

Computer networks have become essential in several aspects of modern society. The performance of information dissemination in networks often determines their overall efficiency. One of the fundamental information dissemination problems is broadcasting. Broadcasting is a process in which a single message is sent from one member of a network to all other members. Inefficient broadcasting could seriously degrade the performance of a network. Therefore, it is of major interest to improve the performance of a network by using an efficient broadcasting algorithm.

Broadcasting is an information-dissemination problem in a connected network, in which one node, called the originator, must distribute a message to all other nodes by placing a series of calls along the communication lines of the network.

(C) A K Peters, Ltd.

|542-795|/08 \$0.50 per page 
Once informed, the informed nodes aid the originator in distributing the message. This is assumed to take place in discrete time units. The broadcasting is to be completed as quickly as possible, subject to the following constraints:

- Each call involves only one informed node and one of its uninformed neighbors.

- Each call requires one unit of time.

- A node can participate in only one call per unit of time.

- In one unit of time, many calls can be performed in parallel.

Formally, any network can be modeled as a connected graph $G=(V, E)$, where $V$ is the set of vertices (or nodes) and $E$ is the set of edges (or communication lines).

Given a vertex $u$ as the originator, we define the broadcast time $b(u)$ of vertex $u$ as the minimum number of time units required to complete broadcasting from vertex $u$. Note that for any vertex $u$ in a connected graph $G$ on $n$ vertices, $b(u) \geq\lceil\log n\rceil$, since during each time unit the number of informed vertices can at most double. The broadcast time $b(G)$ of the graph $G$ is defined as $\max \{b(u) \mid u \in V\}$. The graph $G$ is called a broadcast graph or broadcast network if $b(G)=\lceil\log n\rceil$.

The broadcast function $B(n)$ is the minimum number of edges in any broadcast graph (network) on $n$ vertices. A minimum broadcast graph (mbg), or minimum broadcast network (mbn), is a broadcast graph on $n$ vertices with only $B(n)$ edges. Therefore, an mbg is the cheapest possible broadcast graph architecture (having the fewest edges) in which broadcasting can be accomplished as fast as theoretically possible from any vertex.

The problem of determining $b(u)$ for a vertex $u$ in an arbitrary graph is NPcomplete [Johnson and Garey 79]. The literature on this subject can be divided primarily into two major areas: one on designing approximation algorithms to determine $b(u)$ for a vertex $u$ in an arbitrary graph, the other on designing minimum broadcast graphs.

For the first problem, several approximation algorithms with a polylogarithmic ratio have been suggested [Bar-noy et al. 98, Ravi 94, Elkin and Kortsarz 02]. The best approximation algorithm is presented in [Elkin and Kortsarz 03].

The second problem is also very difficult. The values of $B(n)$ and constructions of mbgs are known only for some small values of $n(n \leq 63), n=2^{p}$, and $n=2^{p}-2$. The broadcast function $B(n)$ was studied in [Farley et al. 79]. The authors showed that hypercubes are mbgs and that $B\left(2^{p}\right)=p 2^{p-1}$ for any $p \geq 1$. 
It was proved independently in [Khachatrian and Haroutunian 90] and [Dinneen et al. 91] that $B\left(2^{p}-2\right)=(p-1)\left(2^{p-1}-1\right)$ for any $p \geq 2$.

Since mbgs seem to be extremely difficult to find, a long sequence of papers presented techniques to construct broadcast graphs and to obtain upper bounds on $B(n)$ (see [Bermond et al. 95, Bermond et al. 92, Dinneen et al. 99, Farley 79, Gargano and Vaccaro 89, Grigni and Peleg 91, Harutyunyan and Liestman 99, Hedetniemi et al. 88, Khachatrian and Haroutunian 90, Knödel 75, Labahn 94]).

However, proving a lower bound that matches the obtained upper bound is extremely difficult. The lower-bound proofs are based on the lower bound on vertex degree. Minimum broadcast graphs on $n=2^{p}$ and $n=2^{p}-2$ vertices are $p$-regular and $(p-1)$-regular graphs, respectively, since in these cases there are matched lower bounds on vertex degree. However, for other values of $n$ the best broadcast graphs are not regular, and so the upper bounds cannot match the lower bounds based on vertex degree.

Several papers have presented methods for constructing broadcast graphs by forming the compound of two known broadcast graphs (see [Bermond et al. 95, Dinneen et al. 99, Harutyunyan and Liestman 99, Khachatrian and Haroutunian 90]). These methods have proven effective for graphs on $n=n_{1} n_{2}$ vertices, from two known broadcast graphs on $n_{1}$ and $n_{2}$ vertices. Broadcast graphs on other sizes can sometimes be formed by adding or deleting vertices from known broadcast graphs (see [Bermond et al. 92]). Unfortunately, there is no effective method for vertex addition or vertex deletion. To obtain a broadcast graph on $n+1$ vertices from a broadcast graph on $n$ vertices, one connects all $n$ vertices to the added vertex. A method based on compounding and merging several vertices into one that allows the construction of the best broadcast graphs for almost all values of $n$, including many primes, is presented in [Harutyunyan and Liestman 99].

In this paper, we describe a more efficient method for vertex addition. To construct a broadcast graph on an odd number of vertices from Knödel graphs (which are the broadcast graphs on an even number of vertices) we add approximately $n / 4$ edges. This result improves the best known upper bound on $B(n)$ for many odd values of $n$, in particular for $n=2^{p}-1$. Our vertex addition method is based on a graph domination set.

Consider the problem of constructing an mbg on $n=2^{p}-1$ vertices. The exact value of $B\left(2^{p}-1\right)$ is known for $2 \leq p \leq 6$. Recently, we showed that $B(127)=389$ [Harutyunyan and $\mathrm{Xu}$ 04]. Some upper and lower bounds on $B\left(2^{p}-1\right)$ are also known in the literature [Farley 79, Harutyunyan and Liestman 99, Labahn 94]. The best lower and upper bounds on $B\left(2^{p}-1\right)$ are as follows: $\left(p^{2}\left(2^{p}-1\right)\right) /(2(p+1)) \leq B\left(2^{p}-1\right) \leq 2^{p-1}\left(p-\frac{1}{2}\right)$. The lower bound is given in [Labahn 94], while the upper bound construction is presented in 
[Harutyunyan and Liestman 99]. See [Hedetniemi et al. 88] and [Fraigniaud and Lazard 94] for a survey on this and related problems.

This paper is structured as follows. In Section 2, we introduce an effective method of vertex addition, and using this method for Knödel graphs we construct a broadcast graph on an odd number of vertices. This construction improves the best known upper bound on $B(n)$ for many values of $n$, including $n=2^{p}-1$. In Section 3 we show that $B(127)=389$ by presenting a minimum broadcast network on 127 vertices.

\section{New Construction Method of Broadcast Networks}

In this section, we introduce an efficient method for vertex addition based on a dominating set. Using this method we will construct new broadcast graphs.

Definition 2.I. Modified Knödel graphs $\mathrm{KG}_{n}=(V, E)$ are defined for even values of $n$. The set of vertices is $V=\{0,1,2, \ldots, n-1\}$, and the set of edges is

$$
E=\left\{(x, y) \mid x \in V, y \in V, x+y=2^{r}-1 \bmod n \text { for } 1 \leq r \leq\lfloor\log n\rfloor\right\} .
$$

If $x+y=2^{s}-1 \bmod n$ for some $s, 1 \leq s \leq\lfloor\log n\rfloor$, then $x$ and $y$ are called $s$-dimensional neighbors.

The modified Knödel graph on $n=2^{p}-2$ vertices is a minimum broadcast graph (minimum broadcast network) [Khachatrian and Haroutunian 90, Harutyunyan and Liestman 99]. First we will find a dominating set of modified Knödel graphs on $n=2^{p}-2$ vertices.

Definition 2.2. In a graph $G=(V, E)$, a set $S \subseteq V$ is called a dominating set if for every $u \in V \backslash S$ there exists a vertex $v \in S$ such that $(u, v) \in E$.

First we will prove the existence of a dominating set of size $2^{p-2}$ in $\mathrm{KG}_{2^{p}-2}$.

Lemma 2.3. Let $S \subseteq V\left(\mathrm{KG}_{2^{p}-2}\right)$ and

$$
S=\left\{2 m \mid 0 \leq m \leq 2^{p-3}-1\right\} \cup\left\{2 m+1 \mid 2^{p-2} \leq m \leq 2^{p-2}+2^{p-3}-1\right\} .
$$

Then $S$ is a dominating set of $\mathrm{KG}_{2^{p}-2}$. 
Proof. Let

$$
\begin{aligned}
S & =S_{1} \cup S_{2}, \\
S_{1} & =\left\{2 m \mid 0 \leq m \leq 2^{p-3}-1\right\}=\left\{0,2,4,6, \ldots, 2^{p-2}-2\right\}, \\
S_{2} & =\left\{2 m+1 \mid 2^{p-2} \leq m \leq 2^{p-2}+2^{p-3}-1\right\} \\
& =\left\{2^{p-1}+1,2^{p-1}+3, \ldots, 2^{p-1}+2^{p-2}-1\right\} .
\end{aligned}
$$

We must prove that for every vertex $x \in V\left(\mathrm{KG}_{2^{p}-2}\right)$ either $x \in S$ or there exists $y \in S$ such that $(x, y) \in E\left(\mathrm{KG}_{2^{p}-2}\right)$.

Suppose $x$ is odd. If $1 \leq x \leq 2^{p-1}$, then $x$ can be expressed as $x=2^{\lceil\log x\rceil}-r$, where $r$ is odd, $1 \leq r \leq 2^{\lceil\log x\rceil-1}-1$, and $0 \leq\lceil\log x\rceil \leq p-1$. Consider the vertex $y=r-1$. Since $x+y=\left(2^{\lceil\log x\rceil}-r\right)+(r-1)=2^{\lceil\log x\rceil}-1$ and $0 \leq\lceil\log x\rceil \leq p-1$, it follows that vertices $x$ and $y$ are connected. From $1 \leq r \leq 2^{\lceil\log x\rceil-1}-1$ it follows that $0 \leq r-1 \leq 2^{\lceil\log x\rceil-1}-2 \leq 2^{p-2}-2$. Thus, $y=r-1 \in S_{1}$, and any odd vertex $x$, where $1 \leq x \leq 2^{p-1}-1$, and vertex $y=2^{\lceil\log x\rceil}-x-1, y \in S$, are $\lceil\log x\rceil$-dimensional neighbors.

All the odd vertices $2^{p-1}+1,2^{p-1}+3, \ldots, 2^{p-1}+2^{p-2}-1$ belong to $S_{2}$, and consequently to $S$.

It remains to consider odd vertices $2^{p-1}+2^{p-2}+1,2^{p-1}+2^{p-2}+3, \ldots$, $\left(2^{p-1}+2^{p-2}\right)+\left(2^{p-2}-3\right)=2^{p}-3$. It is easy to see that $x=\left(2^{p-1}+2^{p-2}\right)+1$ is a neighbor of vertex $y=2^{p-2}-2 \in S_{1}$, since $x+y=2^{p}-1=1 \bmod \left(2^{p}-2\right)$. Similarly, $x=2^{p-1}+2^{p-2}+3$ is a neighbor of $y=2^{p-2}-4 \in S_{1}$, because $x+y=1 \bmod \left(2^{p}-2\right)$. In general, any vertex $x=\left(2^{p-1}+2^{p-2}\right)+(2 s-1)$, where $1 \leq 2 s-1 \leq 2^{p-2}-3$, is a neighbor (first-dimensional neighbor) of $y=2^{p-2}-2 s \in S_{1}$, since $x+y=2^{p}-1=1 \bmod \left(2^{p}-2\right)$.

Therefore, every odd vertex $x$ is a vertex in $S$ or is a neighbor of a vertex in $S$.

Suppose $x$ is even. All even vertices $0,2,4, \ldots, 2^{p-2}-2$ belong to $S_{1}$ and consequently to $S$.

Consider even vertices $2^{p-2}, 2^{p-2}+2, \ldots, 2^{p-2}+\left(2^{p-2}-2\right)=2^{p-1}-2$. Then $y=2^{p-1}+2^{p-2}-1 \in S_{2}$ is the first-dimensional neighbor of $x=2^{p-2}$, since $x+y=2^{p}-1=1 \bmod \left(2^{p}-2\right)$. Similarly, $y=2^{p-1}+2^{p-2}-3 \in S_{2}$ is the firstdimensional neighbor of $x=2^{p-2}+2$, since $x+y=2^{p}-1=1 \bmod \left(2^{p}-2\right)$. In general, the first-dimensional neighbor of $x=2^{p-2}+2 s$, where $0 \leq 2 s \leq 2^{p-2}-2$, is the vertex $y=2^{p-1}+2^{p-2}-2 s-1$, since $x+y=2^{p}-1=1 \bmod \left(2^{p}-2\right)$. Since $0 \leq 2 s \leq 2^{p-2}-2$, it follows that $2^{p-1}+2^{p-2}-2^{p-2}+2-1 \leq y \leq 2^{p-1}+2^{p-2}-1$, and so $y=2^{p-1}+2^{p-2}-2 s-1 \in S_{2}$. For example, $x=2^{p-1}-2$ is a neighbor of $y=2^{p-1}+1 \in S_{2}$, since $x+y=1 \bmod \left(2^{p}-2\right)$.

It remains only to consider the case that $x$ is even and $2^{p-1} \leq x \leq 2^{p}-4$.

We see that $x=2^{p-1}$ is a $(p-2)$-dimensional neighbor of $y=2^{p-1}+2^{p-2}-3$ $\in S_{2}$, since $x+y=2^{p}+2^{p-2}-3=2^{p-2}-1 \bmod \left(2^{p}-2\right)$. Similarly, $x=2^{p-1}+2$ 


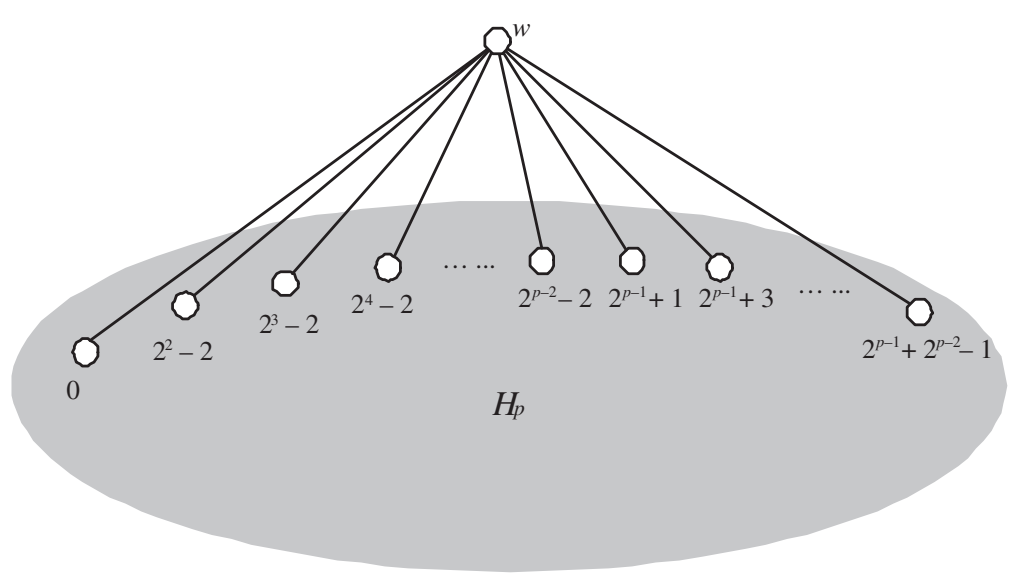

Figure I. Broadcast graph on $2^{p}-1$ vertices.

is a $(p-2)$-dimensional neighbor of $y=2^{p-1}+2^{p-2}-5 \in S_{2}$, since $x+y=$ $2^{p}+2^{p-2}-3=2^{p-2}-1 \bmod \left(2^{p}-2\right)$. In general, any vertex $x=2^{p-1}+2 s$, where $0 \leq 2 s \leq 2^{p-2}-4$, is a $(p-2)$-dimensional neighbor of $y=2^{p-1}+2^{p-2}-3-2 s \in$ $S_{2}$, since $x+y=2^{p-2}-1 \bmod \left(2^{p}-2\right)$.

All even vertices $2^{p-1}+2^{p-2}-2,2^{p-1}+2^{p-2}, \ldots,\left(2^{p-1}+2^{p-2}\right)+\left(2^{p-2}-4\right)=$ $2^{p}-4$ have a $(p-1)$-dimensional neighbor in the set $S$.

The vertex $x=2^{p-1}+2^{p-2}-2$ is a neighbor of $y=2^{p-1}+2^{p-2}-1 \in S_{2}$, since $x+y=2^{p-1}-1 \bmod \left(2^{p}-2\right)$. In general, any vertex $x=2^{p-1}+2 s$, where $2^{p-2}-2 \leq 2 s \leq 2^{p-1}-4$, is a neighbor of $y=2^{p}-3-2 s$ in dimension $p-1$, since $x+y=2^{p-1}-1 \bmod \left(2^{p}-2\right)$. Since $2^{p-2}-2 \leq 2 s \leq 2^{p-1}-4$, we have $2^{p-1}+1 \leq y \leq 2^{p-1}+2 p-2-1$ and thus $y \in S_{2}$.

Therefore, any $x \in V\left(\mathrm{KG}_{2^{p}-2}\right)$ either belongs to the set $S$ or is a neighbor of a vertex in $S$. Thus $S$ is a dominating set.

Now we will present a new method of constructing broadcast graphs on $2^{p}-1$ vertices. The basic idea of this method is to construct a graph on $2^{p}-1$ vertices from the graph $\mathrm{KG}_{2^{p}-2}$ by adding an extra vertex connected with all the vertices of the dominating set $S$ (Lemma 2.3). Consider the graph $G=(V, E)$, with $V=\left\{w, 0,1, \ldots, 2^{p}-3\right\}$ and $E=E\left(\mathrm{KG}_{2^{p}-2}\right) \cup E^{\prime}$, where $E^{\prime}=\{(w, u) \mid u \in$ $\left.S:=\left\{0,2,4,6, \ldots, 2^{p-2}-2,2^{p-1}+1,2^{p-1}+3, \ldots, 2^{p-1}+2^{p-2}-1\right\}\right\}$. The structure of the graph $G$ is shown in Figure 1.

To broadcast from an arbitrary vertex of $\mathrm{KG}_{2^{p}-2}$, all informed vertices call neighbors in dimension $j$ at time $j$ for $1 \leq j \leq p-1$ and neighbors in dimension 1 at time $p$. This is called a dimensional broadcast scheme $(1,2, \ldots, p-1,1)$. We 
will use [Bermond et al. 97, Lemma 3] to describe a minimum-time broadcast scheme in our new broadcast graph.

Lemma 2.4. [Bermond et al. 97] Any cyclic shift of a valid dimensional broadcast scheme is also a valid dimensional broadcast scheme.

In other words, $(i, i+1, \ldots, p-1,1,2, \ldots, i-1, i)$ is a dimensional broadcast scheme in $H_{p}$ for any $i, 1 \leq i \leq p-1$.

Theorem 2.5. $B\left(2^{p}-1\right) \leq 2^{p-1}\left(p-\frac{1}{2}\right)-(p-1)$.

Proof. We will prove that graph $G=(V, E)$ described above is a broadcast graph. To prove this, we must describe a minimum-time broadcast scheme for every originator $u \in V$.

If $u$ is in the dominating set $S$ of $\mathrm{KG}_{2^{p}-2}(u \in S)$, then $u$ informs all other vertices of $\mathrm{KG}_{2^{p}-2}$ according to the dimensional broadcast scheme $(1,2, \ldots$, $p-1,1)$. Since $u$ is idle at time unit $p(u$ informed its 1 -dimensional neighbor at time 1), it sends the message to vertex $w$ at time $p$.

If $u$ is not in the dominating set $S$ of $\mathrm{KG}_{2^{p}-2}\left(u \in V\left(\mathrm{KG}_{2^{p}-2}\right) \backslash S\right)$, there must exist a vertex $v \in S$ that is a neighbor of $u$. Then $u+v=2^{i}-1$ for some $i$, where $0 \leq i \leq p-1$. According to lemma $2.3,(i, i+1, \ldots, p-1,1,2, \ldots, i-1, i)$ is a dimensional broadcast scheme for the originator $u$ in $\mathrm{KG}_{2^{p}-2}$. Under this broadcast scheme, in $p$ time units all the vertices of $\mathrm{KG}_{2^{p}-2}$ will be informed, and vertices $u$ and $v$ will be idle at time $p$ (since $u$ already informed $v$ at time 1 ). Thus, vertex $v$ will inform vertex $w$ at time $p$, since $v \in S$ and it is connected with $w$.

The broadcast scheme from originator $w$ in graph $G$ is more complicated. It is essentially the broadcast scheme of vertex 1 in graph $\mathrm{KG}_{2^{p}-2}$ with minor changes. First consider the dimensional broadcast scheme $(1,2, \ldots, p-1,1)$ from originator 1 in $\mathrm{KG}_{2^{p}-2}$. Recall that under this dimensional broadcast scheme, vertex 1 sends the message to vertex $2^{i}-2$ at any time $i$, where $i=1,2, \ldots, p-1$, and then all vertices $2^{i}-2,1 \leq i \leq p-1$, continue to broadcast according to the dimensional broadcast scheme $(1,2, \ldots, p-1,1)$.

At time $p$, vertices 0 and 1 are idle. Denote this broadcast scheme by $S(1)$. To broadcast in graph $G$ from originator $w$, vertex $w$ sends the message to vertex $2^{i}-2$ at time $i$, where $1 \leq i \leq p-2$, and to vertex $2^{p-1}+1$ at time $p-1$. All vertices $2^{i}-2$, for $i=1,2, \ldots, p-2$, continue the minimum-time dimensional broadcasting $(1,2, \ldots, p-1,1)$ in graph $\mathrm{KG}_{2^{p}-2}$ as in broadcast scheme $S(1)$.

Under the broadcast scheme $S(1)$, vertex $2^{p-1}-2$ receives the message from vertex 1 at time $p-1$, and at time $p$ it informs vertex $2^{p-1}+1$. Now, in the 

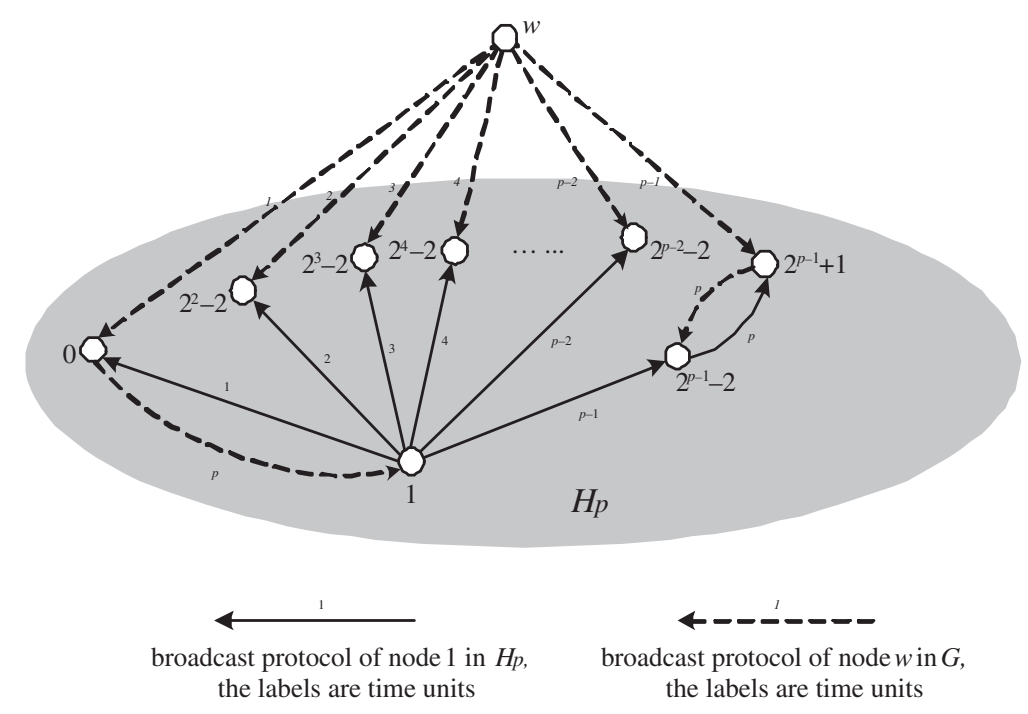

Figure 2. The scheme originating from vertex $w$.

broadcast scheme from originator $w$ in $G$, vertex $2^{p-1}+1$ receives the message from $w$ at time $p-1$ and can inform vertex $2^{p-1}-2$ at time $p$.

At time $p$, all the vertices except vertex 1 will have been informed. Since vertex 0 is idle at time $p$ (vertex 0 already called its neighbor $2^{r}-1$ at time $r$, for $r=2, \ldots, p-1$ ), it can inform vertex 1 at time $p$. Figure 2 illustrates the broadcast scheme from vertex $w$ in $G$. Solid arrows represent the calls in graph $\mathrm{KG}_{2^{p}-2}$ from vertex 1 using the dimensional broadcast scheme $(1,2, \ldots, p-1,1)$. Instead of these calls, the calls designated by the dotted arrows are made in graph $G$ from originator $w$.

Therefore, all vertices of graph $G$ will be informed after time $p$ from any originator. Thus, graph $G$ is a broadcast graph on $2^{p}-1$ vertices. It is obvious that the number of edges in $G$ is equal to

$$
\frac{(p-1)\left(2^{p}-2\right)}{2}+|S|=(p-1)\left(2^{p-1}-1\right)+2^{p-2}=2^{p-1}\left(p-\frac{1}{2}\right)-(p-1) .
$$

So $B\left(2^{p}-1\right) \leq 2^{p-1}\left(p-\frac{1}{2}\right)-(p-1)$.

Previously, the best known upper bound on $B\left(2^{p}-1\right)$ was

$$
B\left(2^{p}-1\right) \leq 2^{p-1}\left(p-\frac{1}{2}\right) .
$$


Although our new upper bound gives only a small improvement, the method of construction is new. The upper bound on $B\left(2^{p}-1\right)$ will be further improved if a dominating set of smaller size can be found in $\mathrm{KG}_{2^{p}-2}$.

Similarly, we can use this method to construct broadcast graphs on any odd number of vertices. To accomplish this, we will add a vertex to a modified Knödel graph.

Consider the graph $G^{\prime}=\left(V^{\prime}, E^{\prime}\right)$, with $V^{\prime}=V\left(\mathrm{KG}_{n-1}\right)=\{w, 0,1, \ldots, n-2\}$ and $E^{\prime}=E\left(\mathrm{KG}_{n-1}\right) \cup E_{0}$, where $E_{0}=\left\{(w, u) \mid u \in S:=\left\{0,2,4,6, \ldots, 2^{p-2}-2\right.\right.$, $\left.\left.2^{p-1}+1,2^{p-1}+3, \ldots, 2^{p-1}+2^{p-2}-1\right\}\right\}$.

Theorem 2.6. $B(n) \leq \frac{n-1}{2}\lfloor\log n\rfloor+2^{\lceil\log n\rceil-2}$.

We omit the proof of this theorem. Our vertex addition method can be applied to graphs that have some properties similar to those of Knödel graphs.

\section{3. $\quad B(127)=389$}

In this section we will show that $B(127)=389$ by constructing a minimum broadcast graph on 127 vertices with 389 edges.

After careful studies of all known mbgs on $2^{p}-1$ vertices, we found that they share a common structure. All the mbgs on $2^{p}-1$ vertices for $p=3,4,6$ have vertices of two types: vertices of degree $p$ and those of degree $p-1$. The number of vertices of degree $p$ is $\left\lceil\left(2^{p}-1\right) /(p+1)\right\rceil$, and the number of vertices of degree $p-1$ is $n-\left\lceil\left(2^{p}-1\right) /(p+1)\right\rceil$.

All the vertices of degree $p-1$ form a cycle of length $n-\left\lceil\left(2^{p}-1\right) /(p+1)\right\rceil$ with some additional chords. Each vertex of degree $p$ is a center of a star, which is connected with $p$ vertices of degree $p-1$. In Figures 3 through 6 the mbgs on $7,15,31$, and 63 vertices are presented.

The mbgs on 7,15 , and 63 vertices have the above-mentioned properties. In [Dinneen et al. 99], another mbg on 15 vertices was proposed (Figure 4(a)). In Figure 4 it is shown that this graph is isomorphic to the one from [Farley et al. 79] (Figure 4(b)). The labeling of the vertices that shows their isomorphism is presented in the figure.

The mbg on 31 vertices proposed in [Bermond et al. 92] uses six pentagons with some additional edges between them (Figure 5(a)). We showed that this graph is isomorphic to the one in Figure 5 (b), which has the properties mentioned above. It has $\left\lceil\frac{25-1}{6}\right\rceil=6$ vertices of degree 5 , and 25 vertices of degree 4 . The vertices of degree 4 form a subgraph that contains a Hamiltonian cycle. These vertices are labeled $0,1, \ldots, 24$. This subgraph on 25 vertices contains 


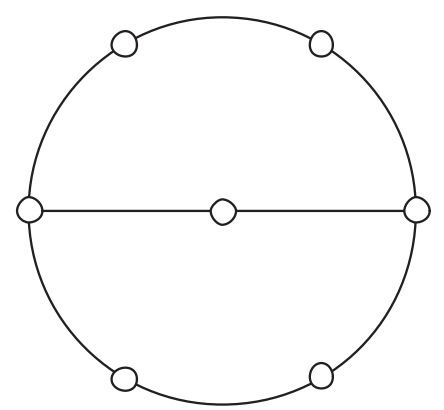

Figure 3. An mbg on seven vertices.

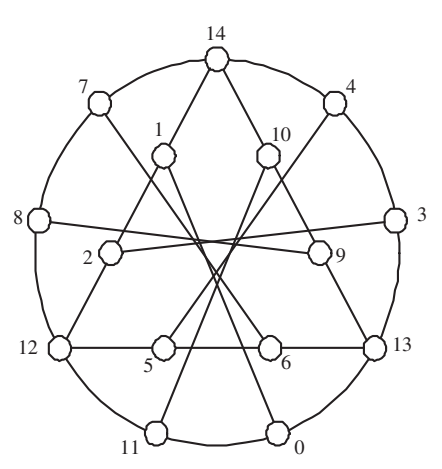

(a)

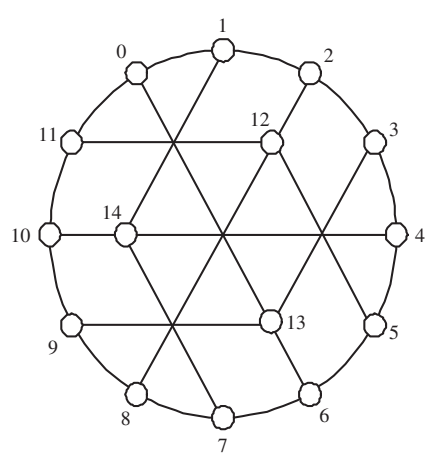

(b)

Figure 4. Isomorphic mbgs on 15 vertices.

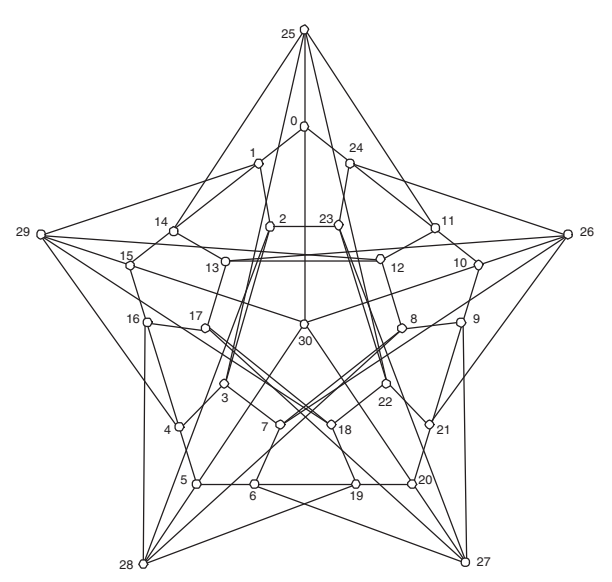

(a)

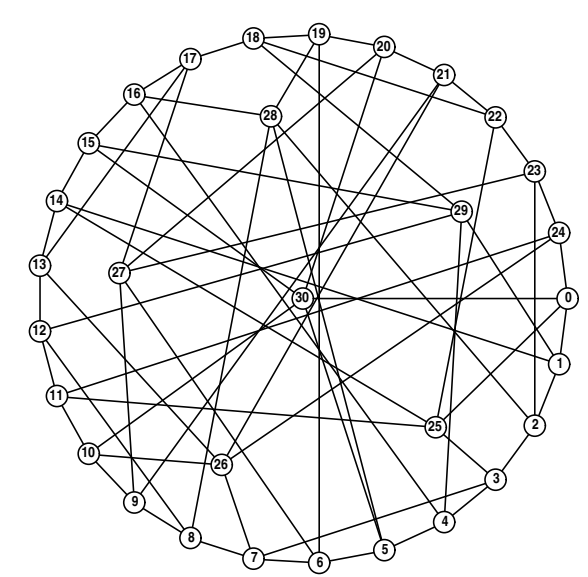

(b)

Figure 5. Isomorphic mbgs on 31 vertices. 


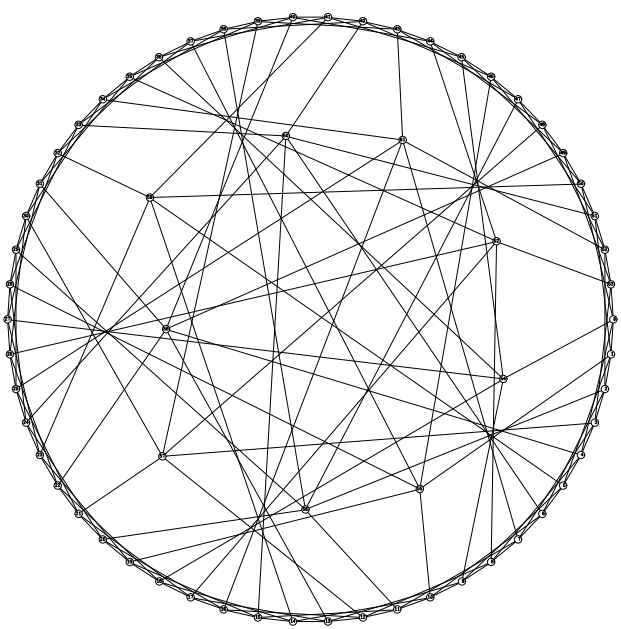

Figure 6. An mbg on 63 vertices.

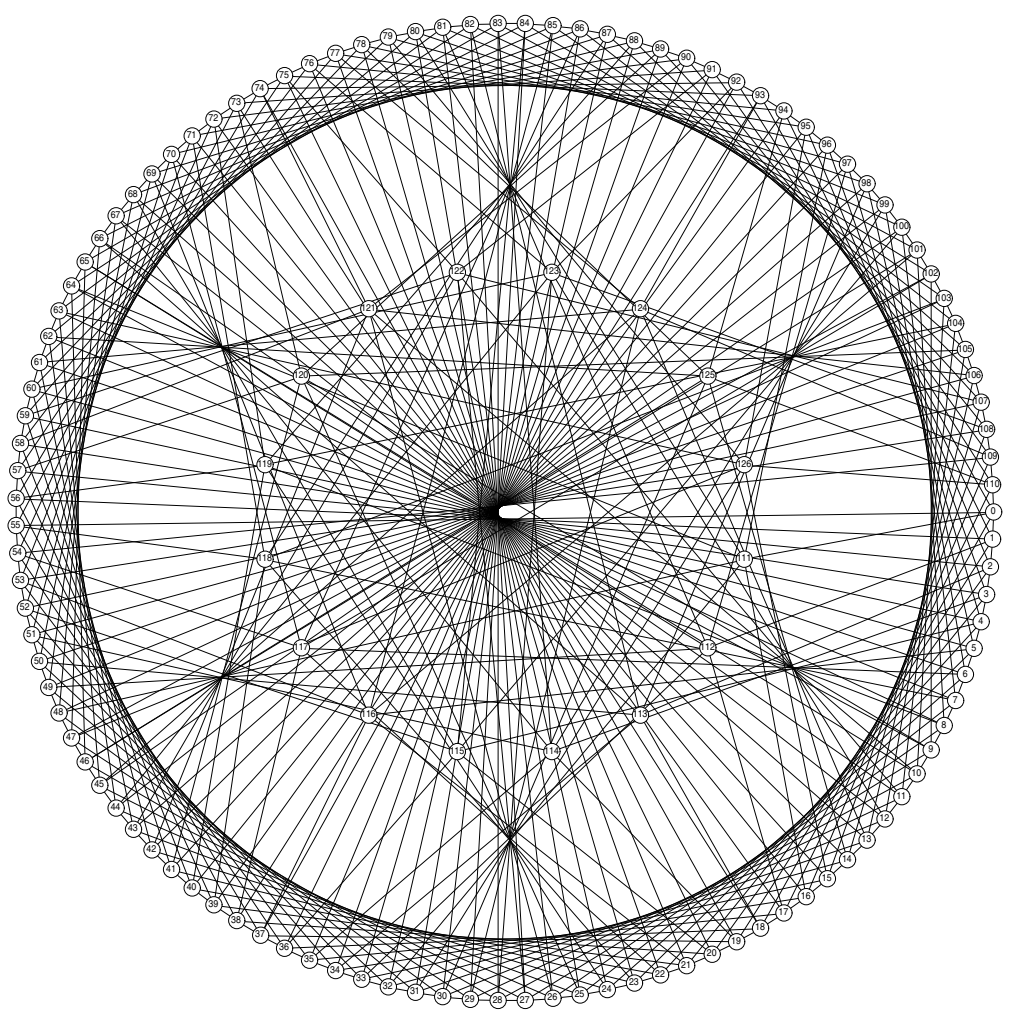

Figure 7. An mbg on 127 vertices. 
some additional chords that connect vertex $i$ to $i+4$ or $i+12$. Also, every vertex of this subgraph is connected to at least one vertex of degree 5 (vertices $25,26,27,28,29,30)$.

All the mbgs on $7,15,31$, and 63 vertices (the mbg on 63 vertices is presented in Figure 6) have the described structural properties, and also,

$$
B\left(2^{p}-1\right)=\frac{p^{2}\left(2^{p}-1\right)}{2(p+1)}
$$

for all $3 \leq p \leq 6$. This led us to the problem of constructing an mbg on 127 vertices having a similar structure.

In [Labahn 94], the lower bound

$$
B\left(2^{p}-1\right) \geq \frac{p^{2}\left(2^{p}-1\right)}{2(p+1)}
$$

is presented. This means that any broadcast graph on 127 vertices with 389 edges is a minimum broadcast graph on 127 vertices. This result was obtained after massive simulations. Although the desired structural properties of an mbg on 127 vertices are known in general, the chords that connect vertices on the Hamiltonian cycle in the subgraph with vertices of degree 6 are very difficult to find. In all previous cases $(p=3,4,6)$, these chords had length either a power of two or the sum of two terms each a power of two.

Even this restriction left us with an extremely large number of cases to consider. Our minimum broadcast graph $G=(V, E)$ is formally described as follows: $V=U \cup U^{\prime}=\{0,1, \ldots, 126\}$, where $U=\{0,1,2, \ldots, 110\}, u \in U$, $\operatorname{deg}(u)=6$, and $U^{\prime}=\{111,112, \ldots, 126\}, u^{\prime} \in U^{\prime}, \operatorname{deg}\left(u^{\prime}\right)=7$. In addition, $E=E_{1} \cup E_{2} \cup E_{3} \cup E_{4} \cup E_{5}$ and $|E|=389$. The subsets $E_{1}, \ldots, E_{5}$ are defined as follows:

- $E_{1}=\{\{0,1\},\{1,2\}, \ldots,\{u, u+1\}, \ldots,\{109,110\},\{110,0\}\}$. These edges form a Hamiltonian cycle of 111 vertices of the set $U$.

- $E_{2}=\{\{0,18\},\{1,19\}, \ldots,\{u, u+18\}, \ldots,\{92,110\},\{93,0\}, \ldots,\{110,17\}\}$. These edges make chords across every 18 vertices on the Hamiltonian cycle.

- $E_{3}=\{\{0,55\},\{1,56\}, \ldots,\{u, u+55\}, \ldots,\{53,108\},\{54,109\}\}$. These edges make chords across half of the cycle.

- $E_{4}=\{\{0,111\},\{1,112\}, \ldots,\{u,(u \bmod 16)+111\}, \ldots,\{110,125\}\}$. These edges connect vertices between $U$ and $U^{\prime}$.

- $E_{5}=\{\{110,126\}\}$. This edge is a complement edge, which connects vertex 110 with vertex 126 . 


\begin{tabular}{l|lllllllllllllllllll}
$i$ & 0 & 1 & 2 & 3 & 4 & 5 & 6 & 7 & 8 & 9 & 10 & 11 & 12 & 13 & 14 & 15 & 16 & 17 & 18 \\
\hline$p(i)$ & - & 0 & 113 & 2 & 59 & 98 & 99 & 25 & 9 & 64 & 9 & 29 & 105 & 124 & 13 & 16 & 111 & 110 & 0 \\
$t(i)$ & 0 & 6 & 6 & 7 & 7 & 5 & 7 & 7 & 6 & 4 & 7 & 7 & 7 & 6 & 7 & 6 & 5 & 7 & 3 \\
$i$ & 19 & 20 & 21 & 22 & 23 & 24 & 25 & 26 & 27 & 28 & 29 & 30 & 31 & 32 & 33 & 34 & 35 & 36 & 37 \\
\hline$p(i)$ & 18 & 75 & 22 & 77 & 118 & 25 & 80 & 27 & 9 & 46 & 124 & 48 & 86 & 111 & 32 & 52 & 36 & 18 & 55 \\
$t(i)$ & 6 & 7 & 7 & 6 & 7 & 6 & 3 & 7 & 5 & 7 & 5 & 7 & 7 & 6 & 7 & 7 & 7 & 4 & 7 \\
$i$ & 57 & 58 & 59 & 60 & 61 & 62 & 38 & 39 & 40 & 41 & 42 & 43 & 44 & 45 & 46 & 47 & 48 & 49 & 50 \\
\hline$p(i)$ & 56 & 59 & 77 & 5 & 43 & 80 & 93 & 38 & 58 & 120 & 43 & 25 & 62 & 27 & 64 & 65 & 111 & 48 & 113 \\
$t(i)$ & 7 & 6 & 5 & 7 & 7 & 5 & 4 & 7 & 7 & 7 & 6 & 5 & 6 & 6 & 5 & 7 & 4 & 5 & 7 \\
$i$ & 51 & 52 & 53 & 54 & 55 & 56 & 63 & 64 & 65 & 66 & 67 & 68 & 69 & 70 & 71 & 72 & 73 & 74 & 75 \\
\hline$p(i)$ & 106 & 115 & 116 & 55 & 0 & 38 & 8 & 111 & 64 & 67 & 49 & 115 & 87 & 117 & 16 & 54 & 18 & 92 & 93 \\
$t(i)$ & 7 & 6 & 7 & 6 & 4 & 6 & 7 & 3 & 6 & 7 & 6 & 7 & 7 & 7 & 7 & 7 & 7 & 7 & 5 \\
$i$ & 76 & 77 & 78 & 79 & 80 & 81 & 82 & 83 & 84 & 85 & 86 & 87 & 88 & 89 & 90 & 91 & 92 & 93 & 94 \\
\hline$p(i)$ & 77 & 124 & 79 & 80 & 111 & 80 & 64 & 101 & 29 & 103 & 117 & 118 & 89 & 120 & 91 & 36 & 93 & 0 & 93 \\
$t(i)$ & 7 & 4 & 7 & 6 & 2 & 7 & 7 & 7 & 6 & 7 & 6 & 6 & 7 & 6 & 7 & 6 & 6 & 2 & 7 \\
$i$ & 95 & 96 & 97 & 98 & 99 & 100 & 101 & 102 & 103 & 104 & 105 & 106 & 107 & 108 & 109 & 110 & 111 & 112 & 113 \\
\hline$p(i)$ & 126 & 111 & 98 & 80 & 98 & 45 & 46 & 84 & 48 & 49 & 120 & 105 & 122 & 15 & 124 & 0 & 0 & 1 & 18 \\
$t(i)$ & 7 & 7 & 7 & 4 & 6 & 7 & 6 & 7 & 6 & 7 & 5 & 6 & 7 & 7 & 7 & 5 & 1 & 7 & 5 \\
$i$ & 114 & 115 & 116 & 117 & 118 & 119 & 120 & 121 & 122 & 123 & 124 & 125 & 126 & & & & \\
\hline$p(i)$ & 19 & 36 & 5 & 38 & 55 & 24 & 25 & 42 & 75 & 44 & 93 & 62 & 110 & & & & \\
$t(i)$ & 7 & 5 & 6 & 5 & 5 & 7 & 4 & 7 & 6 & 7 & 3 & 7 & 6 & & & & \\
&
\end{tabular}

Table I. Broadcast protocol of vertex 0 .

Our mbg on 127 vertices is presented in Figure 7. Since the graph $G$ is not vertex-transitive, the broadcast schemes of different originators are different. Our computer program generated a broadcast scheme that completes broadcasting in seven time units from any originator of $G$. For natural reasons we do not present all the broadcast schemes, all of which are presented in [Harutyunyan and $\mathrm{Xu}$ 04].

In Table 1 we present only the broadcast scheme of vertex 0 . The three rows of the table show that vertex $i$ is informed at time $t(i)$, and it is informed by its parent $p(i)$.

Acknowledgments. The author would like to thank Xiangyang Xu for his help in drawing figures and computer simulations. 


\section{References}

[Bar-noy et al. 98] A. Bar-noy, S. Guha, J. Naor, and B. Schieber. "Multicasting in Heterogeneous Networks." In Proceedings of the Thirtieth Annual ACM Symposium on Theory of Computing, pp. 448-453. New York: ACM Press, 1998.

[Bermond et al. 92] J.-C. Bermond, P. Hell, A. L. Liestman, and J. G. Peters. "Sparse Broadcast Graphs." Discrete Appl. Math. 36 (1992), 97-130.

[Bermond et al. 95] J.-C. Bermond, P. Fraigniaud, and J. Peters. "Antepenultimate Broadcasting." Networks 26 (1995), 125-137.

[Bermond et al. 97] J.-C. Bermond, H. A. Harutyunyan, A. L. Liestman, and S. Perennes. "A Note on the Dimensionality of Modified Knödel Graphs." Int. J. Found. Comp. Sci. 8 (1997), 109-117.

[Dinneen et al. 91] M. J. Dinneen, M. R. Fellows, and V. Faber. "Algebraic Constructions of Efficient Broadcast Networks." In Applied Algebra, Algebraic Algorithms and Error-Correcting Codes 9, Lecture Notes in Computer Science 539, pp. 152-158. Berlin: Springer, 1991.

[Dinneen et al. 99] M. J. Dinneen, J. A. Ventura, M. C. Wilson, and G. Zakeri. "Compound Constructions of Broadcast Networks." Discrete Math. 93 (1999), 205-232.

[Elkin and Kortsarz 02] M. Elkin and G. Kortsarz. "A Combinatorial Logarithmic Approximation Algorithm for the Directed Telephone Broadcast Problem." In Proceedings of the Thirty-Fourth Annual ACM Symposium on Theory of Computing, pp. 438-447. New York: ACM Press, 2002.

[Elkin and Kortsarz 03] M. Elkin and G. Kortsarz. "Sublogarithmic Approximation for Telephone Multicast: Path Out of Jungle." In Proceedings of the Fourteenth Annual ACM-SIAM Symposium on Discrete Algorithms, pp. 76-85. Philadelphia: SIAM, 2003.

[Farley 79] A. M. Farley. "Minimal Broadcast Networks." Networks 9 (1979), 313-332.

[Farley et al. 79] A. M. Farley, S. T. Hedetniemi, A. Proskurowski, and S. Mitchell. "Minimum Broadcast Graphs." Discrete Math. 25 (1979), 189-193.

[Fraigniaud and Lazard 94] P. Fraigniaud and E. Lazard. "Methods and Problems of Communication in Usual Networks." Discrete Appl. Math. 53 (1994), 79-133.

[Gargano and Vaccaro 89] L. Gargano and U. Vaccaro. "On the Construction of Minimal Broadcast Networks." Networks 19 (1989), 673-689.

[Grigni and Peleg 91] M. Grigni and D. Peleg. "Tight Bounds on Minimum Broadcast Networks." SIAM J. Discrete Math. 4 (1991), 207-222.

[Harutyunyan and Liestman 99] H. A. Harutyunyan and A. L. Liestman. "More Broadcast Graphs." Discrete Math. 98 (1999), 81-102.

[Harutyunyan and Xu 04] H. A. Harutyunyan and X. Xu. "Minimum Broadcast Graph on 127 Vertices." Technical Report, Department of Computer Science, Concordia University, Montreal, QC, 2004. Available at http://users.encs.concordia.ca/ $\sim$ haruty/mbg127.pdf. 
[Hedetniemi et al. 88] S. M. Hedetniemi, T. Hedetniemi, and A. L. Liestman. "A Survey of Gossiping and Broadcasting in Communication Networks." Networks 18 (1988), 319-349.

[Johnson and Garey 79] D. Johnson and M. Garey. Computers and Intractability: A Guide to the Theory of NP-Completeness. San Francisco: Freeman, 1979.

[Khachatrian and Haroutunian 90] L. H. Khachatrian and H. S. Haroutunian "Construction of New Classes of Minimal Broadcast Networks." In Proceedings of the Third International Colloquium on Coding Theory, pp. 69-77. Dilijan: Armenian Academy, 1990.

[Knödel 75] W. Knödel. "New Gossips and Telephones." Discrete Math. 13 (1975), 95.

[Labahn 94] R. Labahn. "A Minimum Bbroadcast Graph on 63 Vertices." Discrete Appl. Math. 53 (1994), 247-250.

[Ravi 94] R. Ravi. "Rapid Rumor Ramification: Approximating the Minimum Broadcast Time. Proceedings of the 35th Annual IEEE Symposium on Foundations of Computer Science, pp. 202-213. Los Alamitos, CA: IEEE Press, 1994.

Hovhannes A. Harutyunyan, Department of Computer Science and Software Engineering, Concordia University, Montreal, QC, H3G 1M8, Canada (haruty@cs.concordia.ca)

Received January 25, 2006; accepted May 7, 2007. 